\title{
Intervista a Martino Marangella
}

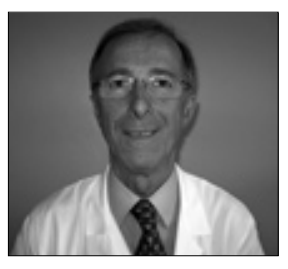

Martino Marangella, MD

Direttore S.C. Nefrologia e Dialisi

A.O. Ordine Mauriziano di Torino

mmarangella@mauriziano.it

Nel centro ove presti attività qual è la percentuale di pazienti monitorati per CKD-MBD stadio 3-5 e quali marker biologici usi a tal fine?

Il nostro Centro segue almeno 800 pazienti con CKD stadio 3-5. Il monitoraggio per CKD-MBD viene esteso in pratica a tutti i pazienti con CKD a partire dallo stadio IV. Gli esami eseguiti di routine sono: Ca-P-ALP-PTH$25 \mathrm{OH} \mathrm{D}_{3} \mathrm{e}$, in casi selezionati, anche $1,25(\mathrm{OH})_{2} \mathrm{D}_{3}$.

Nel centro ove presti attività pratichi il dosaggio della $25(\mathrm{OH}) \mathrm{D}$ e/o della $1,25(\mathrm{OH})_{2} \mathrm{D}_{3}$ ?

Se si, in quale tipo di paziente ed in quale percentuale?

Se no, perché ritieni di non doverla praticare?

Il dosaggio di $25(\mathrm{OH}) \mathrm{D}_{3}$ viene eseguito in quasi tutti i pazienti a partire dallo stadio IV, mentre quello di $1,25(\mathrm{OH})_{2} \mathrm{D}_{3}$ è riservato a casi selezionati (per esempio, PTH inappropriatamente elevato, ipercalcemie, malassorbimenti ecc.).

Applichi la correzione di $25(\mathrm{OH}) D$ in caso di carenza? Perché, con quale protocollo ed in quale tipo di paziente?

In caso di carenza ai pazienti viene prescritto Calcifediolo gtt, in dosaggio variabile in base al peso, alla stagione, al grado di carenza. In alcuni casi usiamo colecalciferolo o ergocalciferolo settimanale o mensile. Vengono trattati tutti i pazienti carenti o insufficienti.

Nel centro ove presti attività in quale percentuale o in quali casi clinici particolari pratichi la biopsia ossea?

Il nostro Centro ha abbandonato la pratica della biopsia ossea. Questa viene eseguita in casi molto particolari.
Nel centro ove presti attività in quale percentuale e in quali stadi di CKD usi indagini strumentali per la ricerca delle calcificazioni vascolari? Quali indagini utilizzi?

A partire da stadio IV-V si programma Rx Lombare Laterale con misura della calcificazione aortica in L1-LA. Quasi tutti i pazienti eseguono Ecocardiogramma e pazienti con patologia vascolare Ecodoppler TSA.

Nel centro ove presti attività quale è la percentuale di pazienti in trattamento sostitutivo che resta nel range di normalità (secondo le linee guida SIN) per Ca, P e PTH?

Fra il 10 e il 15\% per tutti i parametri. Fra 50-60\% per la fosforemia.

Nel centro ove presti attività qual è la percentuale dei vari chelanti usati per ricercare il controllo del Ca-P nei pazienti in trattamento sostitutivo (anche in off-label treatment)?

Chelanti a base di calcio circa $40 \%$, in associazione con Sevelamer 30\%. Sevelamer da solo 18\%. 10-12\% circa con Foznol.

Nel centro ove presti attività qual è la percentuale di pazienti in CKD 3-5 sottoposta a dieta ipoproteica per il controllo della iperfosforemia?

Non superiore al 25\%.

Nel centro ove presti attività quale percentuale di pazienti in trattamento sostitutivo assume vit-D o analoghi, calciomimetico, o entrambi?

Vitamina D o analoghi: $66 \%$ di cui 25\% Paracalcitolo i.v. Cinacalcet $12 \%$, quasi sempre in associazione con vitamina $\mathrm{D}$.

Le calcificazioni vascolari-evento davvero comune nel paziente con CKD-sono associate ad un elevato rischio di mortalità in dialisi ma non esistono studi d'intervento che dimostrino chiaramente che la loro riduzione migliori la sopravvivenza. Come va interpretato tale apparente paradosso?

Studi controllati aventi per oggetto il rapporto fra calcificazioni vascolari e mortalità in dialisi mancano a mio parere per i seguenti motivi:

1. le metodiche di imaging oggi disponibili per la 
valutazione dell'andamento delle calcificazioni sono, o troppo grossolane o troppo complesse per prestarsi ad uno studio prospettico;

2. molti pazienti giungono all'osservazione del nefrologo presentando già calcificazioni vascolari importanti;

3. poiché la progressione delle calcificazioni è un processo lento, uno studio prospettico dovrebbe avere una durata molto lunga, e inoltre un arruolamento molto numeroso;

4. l'outcome "mortalità" nella popolazione in dialisi risente di un numero molto elevato di co-variabili che hanno un effetto confondente sui risultati. Anche questo richiede un arruolamento molto ampio ed un finanziamento consistente;

5. le problematiche attinenti le calcificazioni vascolari coinvolgono la fosforemia e i chelanti. È dimostrato che la compliance di lungo periodo ai chelanti non raggiunge il 50\% e di conseguenza, il raggiungimento ed il mantenimento di fosforemie in target non è facilmente ottenibile nella maggior parte dei pazienti.

Del cosiddetto effetto 'pleiotropico' della vit-D e dei suoi analoghi, quanto e cosa è applicabile alla CKD-MBD?

È noto ed accettato che la vitamina $\mathrm{D}$ svolge molte azioni diverse da quelle direttamente connesse con il metabolismo minerale, genericamente definite "pleiotropiche". Molti studi di associazione nella popolazione uremica hanno documentato un maggior rischio di mortalità sia generale che da causa cardio-vascolare nei pazienti con livelli più bassi di vitamina $\mathrm{D}$, sia $25(\mathrm{OH}) \mathrm{D}_{3}$ che $1,25(\mathrm{OH})_{2} \mathrm{D}_{3}$.

Questo vale sia per i metaboliti attivi della vitamina D - calcitriolo e paracalcitolo, meglio definiti vitamin D receptor activators o VDRA - sia per la $25(\mathrm{OH}) \mathrm{D}_{3}$ la cui conversione a $1,25(\mathrm{OH})_{2} \mathrm{D}_{3}$ a livello tissutale potrebbe svolgere un ruolo autocrino/paracrino.

Fra gli effetti non minerali dei metaboliti attivi della vitamina $\mathrm{D}$, vi è quello antireninico diretto, che si esplica con un meccanismo genico di inibizione della trascrizione della renina a livello tissutale. Questo effetto antireninico è di grande utilità e sinergico con la terapia con ACEi e/o sartanici che, come è noto, inducono un aumento dei livelli di renina.

Gli effetti anti-infiammatori dei VDRA, mediati da una inibizione di NF-kB, e quelli anti-fibrogenici, si esplicano anche a livello cardiaco, traducendosi in una prevenzione e regressione della ipertrofia ventricolare sinistra e in un miglioramento di struttura e funzione cardiaca. Pertanto, la combinazione degli effetti anti-reninico infiammatorio e fibrogenico, esplicantesi a livello cardiovascolare, potrebbe spiegare il rapporto inverso fra vi- tamine D e mortalità. Si deve comunque sottolineare che molti dei dati fin qui disponibili derivano da studi in vitro o su animali transgenici, mentre i dati pre-clinici sull'uomo sono ancora molto scarsi.

Inoltre, anche su questo argomento mancano studi di intervento centrati sull'hard outcome "sopravvivenza".

\section{1-84; 1-34; 36-84; 7-84: quale paratormone per quale re- cettore e per quale paziente?}

Le problematiche relative al dosaggio del PTH sono esplose in ambito nefrologico alla fine del secolo scorso. I primi anni del III millennio non hanno portato ad una completa definizione della materia. Semplificando, la mancanza di uno standard accettato a livello internazionale fa sì che ad oggi i valori di riferimento debbano essere adattati allo specifico kit di dosaggio usato. Pertanto le conclusioni che possiamo trarre ad oggi sono le seguenti:

a. i singoli metodi hanno tutti una buona affidabilità e correlano molto bene fra loro;

b. le differenze fra valori assoluti impongono di costituire valori di riferimento specifici;

c. pertanto, le soglie decisionali ed i target ottimali dovrebbero essere riferiti agli intervalli di riferimento ed il confronto fra metodi dovrebbe utilizzare fattori di correzione basati sulla pendenza delle rette di regressione calcolate;

d. le aziende produttrici dovrebbero dichiarare ed il clinico dovrebbe sapere, quale PTH venga riconosciuto e dosato e quali le interferenze attese;

e. è prevedibile lo sviluppo di metodi, non utilizzanti traccianti radioattivi, per il dosaggio del PTH 1-84 ed eventualmente di frammenti C-term, N-troncati in posizione 1-7, ritenuti già oggi importanti dal punto di vista fisiopatologico.

$\grave{E}$ pensabile che ci ritroveremo a curare la proteinuria con una 'semplice' vitamina (la D od $i$ suoi analoghi)?

Ritengo che questa ipotesi potrà trovare conferma, almeno parziale in futuro.

Ho accennato sopra ai molti dati sperimentali che evidenziano tre azioni dei VDRA potenzialmente utili sia nell'interferire con la progressione dell'insufficienza renale, che nel ridurre la proteinuria. Gli effetti anti renina, anti-infiammazione ed anti neofibrogenesi, costituiscono la premessa per sperimentare, anche in ambito clinico, l'efficacia dei VDRA.

I dati ad oggi disponibili su pochi studi pre-clinici e su uno multicentrico(VITAL), sembrano indicare un qualche effetto aggiuntivo dei VDRA se usati in associazione con ACEi e/o sartanici nelle nefropatie proteinuriche. 
Dunque a mio parere, le molte promesse basate su studi sperimentali attendono di essere mantenute dalla sperimentazione clinica su casistiche ampie e ben controllate.

\section{Quale il fattore di rischio nella CKD-MBD di più difficile ap- proccio?}

Non sarà inutile ribadire che il fattore chiave è la fosforemia per motivi ben noti che comprendono la difficoltà di riduzione dell'intake dietetico, la scarsa efficienza delle metodiche dialitiche, con l'eccezione forse della dialisi notturna, la scomodità della terapia cronica con $\mathrm{i}$ chelanti, la perdurante incertezza su quali siano i valori di sicurezza per i pazienti. In quasi tutti gli audit cui ho partecipato o di cui ho letto i resoconti, una elevata percentuale di pazienti, mai inferiore al $30-40 \%$, non riesce a raggiungere e a mantenere valori target di fosforo. A mio parere il fattore critico è la compliance alla terapia, fortemente condizionata dalla necessità di assumere un certo numero di pillole, a volte troppe, ai pasti. L'assenza di conseguenze cliniche percepibili dal paziente, nel breve-medio periodo, fa mancare alla terapia l'effetto di scoraggiarne la sospensione. Allora, la nostra è una lotta senza speranza? Forse no, se l'industria farmaceutica riuscirà a metterci a disposizione farmaci più efficaci, più semplici da assumere, magari in forma di una pillola al giorno, da prendere al mattino. Un'ipotesi potrebbe essere quella di trovare un inibitore specifico del trasporto intestinale del fosforo, ad esempio un inibitore del carrier intestinale sodio dipendente, denominato $\mathrm{Npt}-2 \mathrm{~b}$.

\section{L'acidosi metabolica ha un ruolo nella CKD-MBD o quan- tomeno nella Osteodistrofia Uremica?}

Pur essendo noto da anni che l'acidosi metabolica cronica interferisce con il metabolismo scheletrico, questo argomento viene poco frequentato in ambito nefrologico. Eppure l'uremico diviene presto un paziente in acidosi metabolica cronica. L'effetto dell'acidosi sull'osso è in primis fisico-chimico, in quanto l'aumento della concentrazione protonica produce nell'osso dissoluzione della idrossiapatite, con ciò contribuendo a limitare severità e progressione della acidosi. In una seconda fase l'effetto è cellulo-mediato, in quanto l'acidosi stimola l' attività osteoclastica ed inibisce quella osteoblastica.

Nelle fasi meno avanzate di insufficienza renale all'effetto sull'osso della acidosi, si aggiunge quello renale con un aumento della calciuria e un peggioramento del bilancio esterno del calcio.

È pertanto importante, anche nella IRC stadio 3-4, dedicare attenzione alle problematiche dell'acidosi, modulando la terapia, ad esempio nella scelta dei chelanti, anche su questo target. Anche la scelta del tipo di acque, come la prescrizione di acque minerali bicarbonato-calciche, può avere effetti benefici significativi sulla salute dell'osso.

\section{Commento conclusivo}

E stato scritto e molte volte ribadito che le alterazioni del metabolismo minerale contribuiscono per circa il $10 \%$ al rischio cardiovascolare in corso di CKD. Si tratta di una quota non trascurabile ed importante, in quanto suscettibile di intervento terapeutico da parte del nefrologo. La nefrologia si è data pertanto dei target entro $\mathrm{i}$ quali i pazienti con $\mathrm{CKD}$ dovrebbero rientrare e verso $\mathrm{i}$ quali gli interventi terapeutici sono mirati.

Tuttavia, ad una premessa rassicurante si contrappone una serie di problemi. Vediamo quali. L'intervallo ottimale dei parametri di metabolismo minerale è stabilito in modo arbitrario, mediandolo dai risultati di studi osservazionali più o meno ampi: questo non garantisce che i target consigliati siano davvero quelli ottimali (vedi fosforemia). Per alcuni parametri abbiamo problemi di affidabilità del dosaggio e questi si riflettono sulla interpretazione delle correlazioni clinico-laboratoristiche (vedi PTH). La percentuale di pazienti che riescono a raggiungere e a mantenere tutti i target consigliati è lungi dall'essere soddisfacente (valori intorno al 10\%). Gli strumenti terapeutici di cui disponiamo non sono ancora soddisfacenti (chelanti del fosforo), spesso costosi o con problemi di tollerabilità nel lungo periodo. Molti pazienti quando giungono alla osservazione del nefrologo presentano già importanti alterazioni dell'apparato cardiovascolare, la cui reversibilità è spesso improbabile.

Ultimo, ma non per importanza, la motivazione del nefrologo, a volte non sufficientemente aggiornato sull'argomento, altre volte disarmato di fronte alla difficoltà di gestione di una patologia cronica, oppure scettico sulle possibilità di modificare l'outcome dei pazienti. Rispetto a questo ultimo punto siamo di fronte ad un problema oggettivo, poiché gli studi controllati di intervento sono, o non disponibili, o deboli dal punto di vista statistico, o criticabili nel disegno e nella conduzione.

Se quanto esposto può rappresentare il pessimismo della ragione, l'ottimismo della volontà si esprime attraverso una formidabile mole di studi sull'argomento, tale da contribuire a mantenere il grande fascino che la materia esercita sulla comunità nefrologica. 